\title{
CONGENITAL BOWING OF THE LONG BONES: REPORT OF A CASE
}

\author{
BY \\ A. D. BAIN and HELEN S. BARRETT \\ From the Departments of Pathology and Anatomy, University of Edinburgh, and the Royal Hospital for Sick Children, \\ Edinburgh
}

(RECEIVED FOR PUBLICATION MAY 8, 1959)

Congenital bowing of many long bones in a single individual is comparatively rare and Angle (1954), in a review of the English literature, found only eight reported cases. On the other hand, bowing of individual long bones occurs with greater frequency, the same author reviewing 12 cases of anterior and 14 cases of posterior tibial angulation and three cases with bilateral involvement of the femora only. The present case is that of a stillborn child who had congenital bowing of several long bones. In order to ascertain the presence or absence of accompanying abnormalities, anatomical and radiological investigations were undertaken, as detailed dissection of the entire skeleton had not previously been reported in these cases.

The diagnosis in this case was based on the presence of typical deformities in the lower limbs, associated with cutaneous dimpling.

\section{Case Report}

History. The mother, aged 23, had been well during this pregnancy, and was first seen one month before the estimated date of delivery when she was found to have an acute hydramnios. Radiological examination failed to reveal foetal malformation, but foetal ascites or hydrops foetalis was suspected. The pregnancy continued and the mother was delivered of a mature stillborn female foetus weighing $3,175 \mathrm{~g}$. The mother's blood group was $\mathbf{O}, \mathbf{R h}+$. Blood Wassermann was negative.

Previous maternal history showed that the only notable illness was two attacks of pneumonia contracted at 3 and 4 years of age, but since then she had experienced no trouble with her chest. She had been pregnant on two previous occasions: in 1948 she had a spontaneous delivery of a full-term healthy male infant weighing $8 \mathrm{lb} .12 \mathrm{oz} .(3,968 \mathrm{~g}$.$) ; this child is now alive and well. In$ 1951 she became pregnant again, but aborted spontaneously at the sixth week.

The family history is clear. She has two brothers and one sister, none married and all in good health. There is no history of any babies suffering from congenital abnormalities in either her own or her husband's family.
External Appearance. The lower limbs appeared relatively short in comparison with the trunk and arms (Fig. 1). There was pronounced anterior angulation of the legs immediately above the ankles. At the apex of the protuberance there was a clearly defined cutaneous dimple. The feet showed a bilateral talipes equino-varus. No abnormality was noted in the upper limbs. The head was unusual in shape with considerable protuberance of

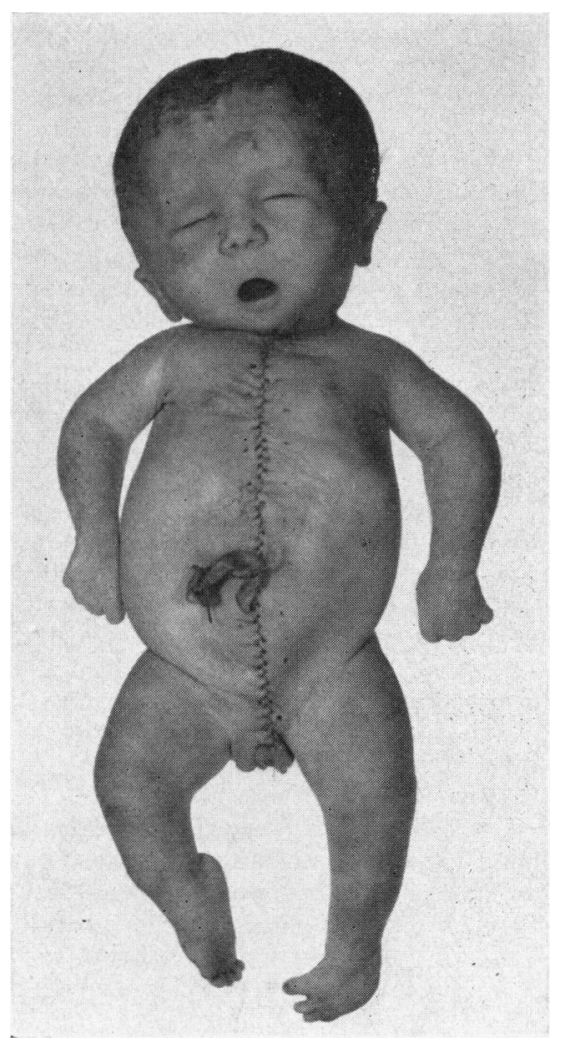

FIG. 1.-Photograph at autopsy showing relative shortness of lower limbs with anterior angulation of tibiae. Note shape of head. 
the frontal bones and a widely patent frontal suture. In view of these abnormalities the baby was examined radiologically before the autopsy (Fig. 2).

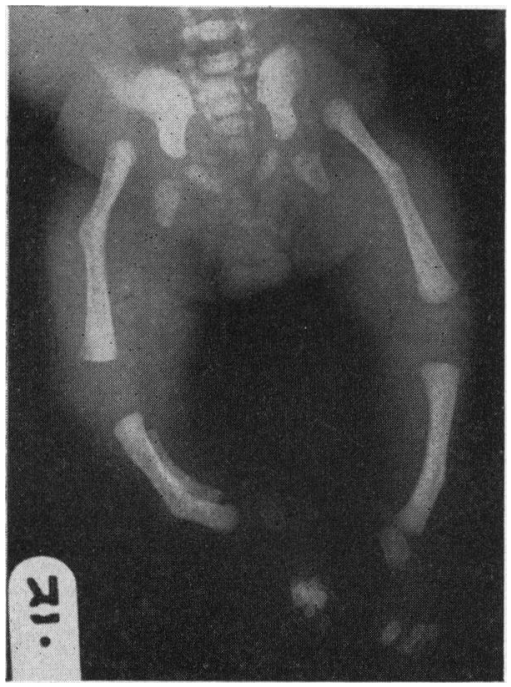

Fig. 2.-Radiograph of lower limbs showing anterior angulation of bones and splaying of ischia.

Autopsy Findings. The subcutaneous tissues were slightly oedematous, and all the serous sacs contained an excess of free fluid. The brain was injected with formalin in order to leave the skull intact for dissection and, when removed at a later stage, showed no pathological abnormality. The pharynx, oesophagus and trachea were normal. The lungs were completely atelectatic and were slightly compressed as a result of the excess free fluid in the pleural sacs. No subpleural haemorrhages were present. The heart, thyroid and thymus showed no abnormality. The alimentary tract was normal. The liver was slightly enlarged and paler than normal but showed no other abnormality. The Prussian blue reaction was negative. The gall bladder, bile ducts, pancreas, spleen, adrenals, ovaries, tubes and uterus showed no pathological changes. The right kidney was enlarged to about one and a half times the normal size on account of a hydronephrosis. The right ureter was moderately dilated, but there was no obstruction at either the pelvi-ureteric or vesico-ureteric junctions to explain the hydronephrosis. The left kidney and left ureter were normal. No abnormality was noted in the bladder or urethra.

Microscopical Findings. The lung parenchyma was mature; the alveoli contained an excess of amniotic débris, compatible with an anoxial state. The liver showed a considerable excess of erythropoiesis, much of this being of primitive type. In the spleen, groups of normoblasts were present throughout the pulp, showing excessive erythropoiesis for a mature foetus. The pancreas, adrenals and heart were normal. The kidneys were mature. Sections were taken from rib, sternum, tibia and fibula. In all of these there was a normal alignment of cartilage cells at the epiphyseal junction, and normal ossification in these regions. Sections of the placenta and membranes failed to reveal any pathological condition.

Further Investigation. Immediately following the autopsy the body was immersed in formalin. The right lower limb together with the ischium and conjoint ramus was amputated for histological examination. After decalcification, serial sagittal sections were made from the right femur and sections were also taken from the right tibia. All were stained with haematoxylin and eosin but fixation was poor and the results were disappointing. The remainder of the body was macerated in potassium hydroxide. The joints of the pelvis, left hip and knee were left intact, as were those of the right shoulder and elbow and most of the vertebral column; the left upper limb and several cervical vertebrae were macerated further to provide dried and separate bones. Radiographs were taken at many stages.

After maceration in potassium hydroxide cartilage remains gelatinous. In this foetus some of the 'cartilage' did not have this gelatinous quality and is described as 'membrane'. Histological examination of this tissue was not possible.

The long bones of the left lower limb and the right humerus were then sectioned longitudinally with a fretsaw and their architecture was compared with that of macerated bones from six normal stillborn foetuses. In the abnormal bones the sections passed through the nutrient foramen and through the convexity of the angulation. The normal bones were cut in similar planes. The bones of the lower limb were thus sectioned in a sagittal plane and the humeri in a coronal plane. When the sections were examined under a hand lens, the direction of the trabeculae which lay longitudinally in the plane of section was easily seen as the trabeculae were outlined by the intervening vascular spaces.

Examination of Skeleton. The bones were all smaller and lighter than normal. The abnormalities noted were bilaterally symmetrical except where otherwise stated. Epiphyseal centres of ossification for the lower ends of the femora and the upper ends of the tibiae were absent. The long bones showed multiple small vascular foramina towards the ends of the shafts. There was no evidence of fracture.

HEAD. A cleft soft palate extended into the posterior part of the hard palate. The base of the skull (chondrocranium) was small in proportion to the vault. The interfrontal suture was wide open and the frontal bones were bulging. The cranial capacity was within normal limits. The individual bones were normal.

Vertebral Column. There were seven cervical, 12 thoracic, six lumbar and four sacral vertebrae. The cervical column was flexed, this flexion being acute between $\mathrm{C} 3$ and $\mathrm{C} 4$. No bony cause for this flexion was found.

Spina Bifida. Spina bifida was present in the cervical region (C2-C6) and in the lumbosacral region from $\mathrm{Ll}$ 
downwards. The laminar defect was gross in the lumbosacral region but was less marked in the cervical region. The defects were bridged across by membrane. The bony laminae of $\mathrm{C} 7$ and of $\mathrm{T} 1$ had met in the midline and undergone premature synostosis.

Abnormality of Pedicles (Fig. 3). The pedicles in the lumbar region were ossified. The pedicles in the thoracic and cervical column were not ossified but represented by membranous bars uniting the bony neural arch to the body. Each bony neural arch was made up of a lamina with superior and inferior articular processes. The right pedicle of T11 was partly ossified, a spicule of bone being continued forwards from the bony neural arch but failing to reach the body, to which it was attached by membrane. The right pedicle of T12 was almost completely ossified. On the left side the transition from membranous to bony pedicle took place in the same manner but occurred one segment lower.

Neuro-central Joints. These were clearly defined in the lumbar region where the bony neural arches continued forwards beyond the pedicle to form the lateral parts of the body, leaving a normal neuro-central cartilaginous joint between bony neural arch and centrum.

In the cervical and thoracic regions no typical neurocentral joints were present. The cervical bodies were transversely elongated; $\mathrm{C} 3$ and $\mathrm{C} 4$ showed a small accessory centre of ossification in the right lateral part of the body, and in C6 a knob of bone was incompletely fused to the right side of the body. The thoracic bodies were heart-shaped and transversely elongated. These findings suggested that ossification was present in the anterior ends of some of the neural arches and that the neuro-central synchondroses had undergone premature synostosis in $\mathrm{C} 6$ and possibly in the thoracic region as well.

Transverse Processes. These were normally ossified in the lumbar region and in the thoracic region where they formed the most anterior part of the bony neural arches. In the cervical region the transverse processes were membranous except in $\mathrm{C} 7$ where the transverse element was bony and continuous with the bone forming the upper and lower articular processes. In this vertebra the left costal element was membranous while the right costal element formed the upper head of a two-headed first right rib.

SACRUM. The bodies and pedicles of the sacral vertebrae were normal. The cartilaginous lateral masses were small, thus producing a very narrow sacrum. No centres of ossification were present in the lateral masses.

Pelvis (Fig. 4). The inlet of the pelvis was abnormally long antero-posteriorly and narrow transversely. The decrease in transverse diameter was partly accounted for by the narrowness of the lateral masses of the sacrum. The antero-posterior diameter of the brim was almost twice the greatest transverse diameter.

The outlet of the pelvis was extraordinarily wide. The remaining (left) ischium was splayed forwards and laterally so that the conjoint ramus ran medially to the pubis. This conjoint ramus was membranous.

A normal sacro-iliac joint cavity was present on both sides. The left hip was dislocated, the false socket being

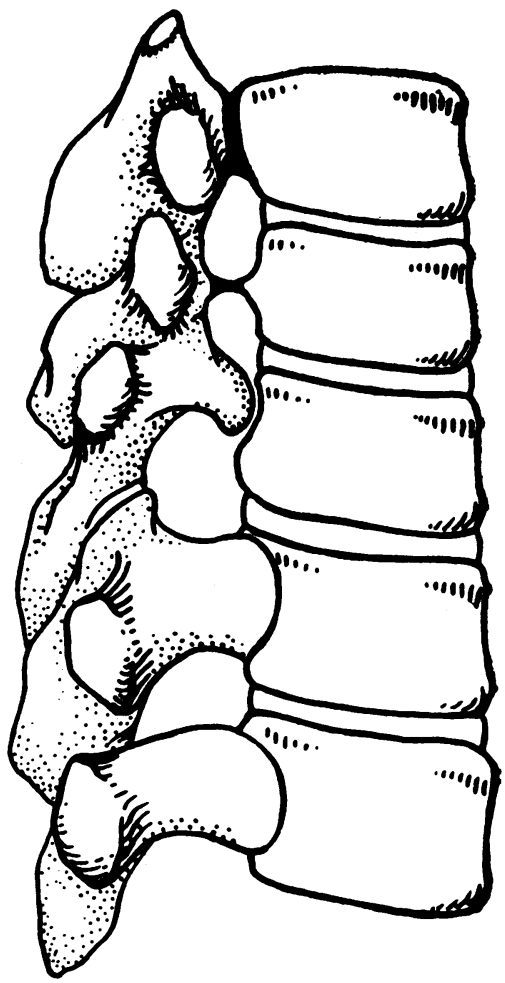

Fig. 3.-Drawing of lateral view of T.10-L.2 showing normal pedicles and neuro-central joints in L.1 and L.2. Pedicle of T.10 is membranous and neuro-central joint is absent. Pedicle of T.11 is partly ossified and pedicle of T.12 almost completely ossified.

above and slightly anterior to the acetabulum. The acetabular rim was normal.

LeFt Femur (external form) (Fig. 5). A spine projected forward from the junction of the upper and middle thirds of the shaft. This was the most obvious abnormality. The spine was not attached to the skin and no dimple was present. At the level of the spine the shaft was bent slightly forwards. Above and below the angulation the shaft was straighter than usual, the normal anterior convexity of the shaft being absent. The ends of the shaft were normally expanded. The only nutrient artery entered the upper third of the back of the bone and was directed distally and forwards towards the apex of the angulation.

LefT AND Right Femora (internal structure) (Fig. 6). At the site of angulation the cortical bone was greatly thickened posteriorly and composed of trabeculae converging towards the spine. The nutrient artery ran along the upper of these radiating trabeculae and was directed towards the apex and the angulation. The marrow cavity was displaced anteriorly by this cortical thickening. The cortical bone forming the spine was of normal thickness.

Histological examination of the shaft of the femur (Fig. 7) showed that some bony trabeculae had an acel- 


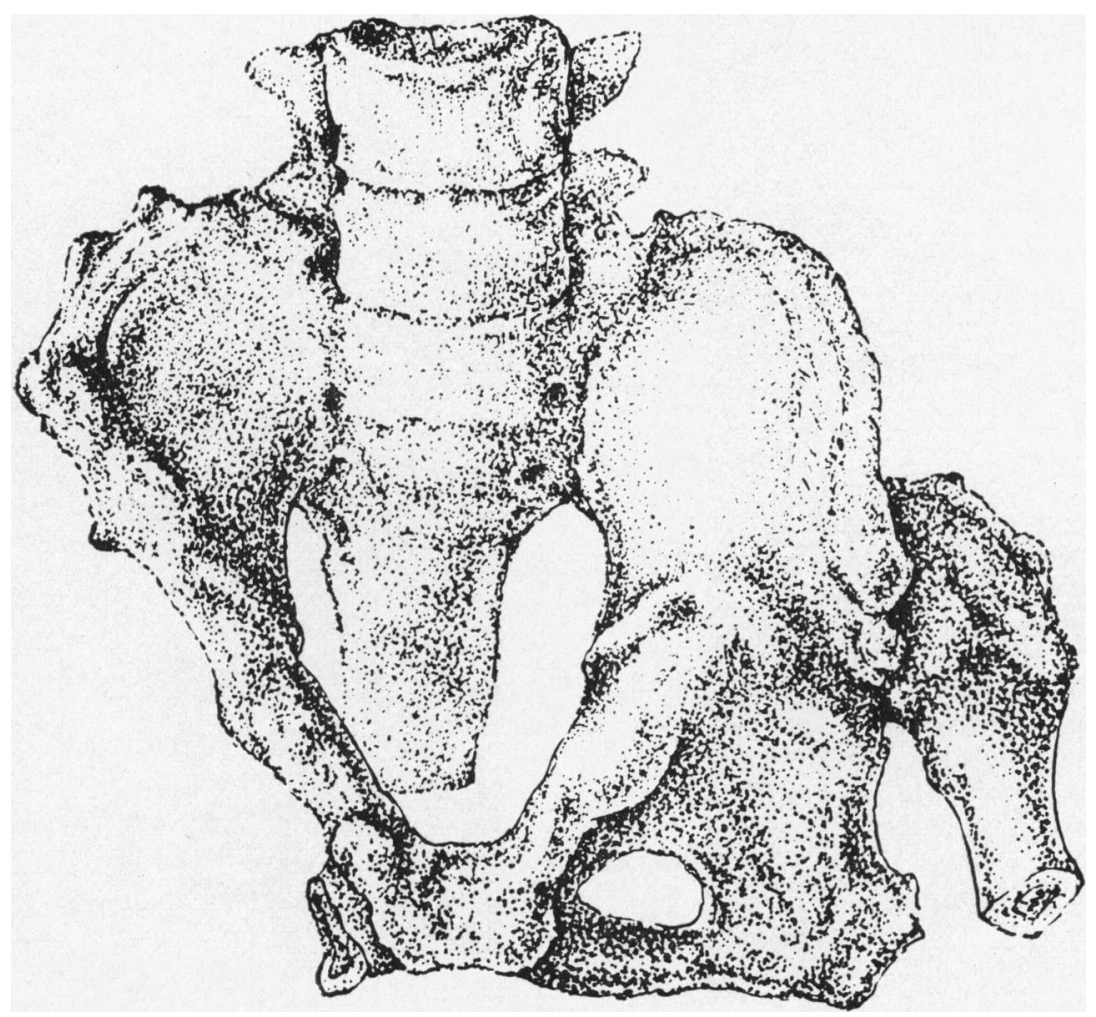

Fig. 4.-Pelvis drawn to scale with L.4, 5 and 6 attached and right ischium removed. Note abnormal shape of pelvic brim; narrowness of lateral masses of sacrum (identifiable on left side between first anterior sacral foramen and sacro-iliac joint); left ischium splayed forward and laterally; dislocation of left hip.

lular cartilaginous core. These cartilaginous cores were more numerous in trabeculae above and below the angulation, more numerous anteriorly than posteriorly, and towards the medullary cavity. At the angulation no cartilage was present in the trabeculae converging towards the spine though cartilage was present in some trabeculae near the medullary cavity in the region of the spine. Subperiosteal trabeculae showed cartilage cores only at the anterior part of the middle of the shaft. The compact bone of the shaft showed many scattered areas of primitive bone, i.e. areas where bone cells were numerous and rounded and separated by a relatively small amount of matrix. The matrix showed lines of irregular density, most numerous in the region of the spine. In the shaft of the bone there were remarkably few osteoblasts and osteoclasts were virtually absent. There were no osteoclasts in the spine. At the metaphysis the bone structure was normal. The periosteum was intact in median sections. The outer fibrous layer was thin over the apex of the spine but normal elsewhere. The inner cellular layer was abnormally thin above and below the spine and absent over the spine where the fibrous layer was in direct contact with the bone. Both layers of periosteum were normal towards the metaphysis and over the concave aspect of the angulation.

LEFT TIBIA (Fig. 5). This showed pronounced anterior angulation at the junction of the middle and lower thirds of the shaft. The apex of this angulation was on the anterior border of the tibia, and the periosteum here was attached by tough fibrous tissue to the overlying skin dimple. The shaft of the tibia above and below the angulation was less curved than normal. As in the femur, the cortical bone on the posterior aspect of the shaft was greatly thickened and composed of trabeculae converging towards the apex of the angulation, this thick cortex narrowing and displacing the medullary cavity forwards. The cortical bone forming the apex of the angulation was slightly thickened. The canal for the nutrient artery ran in the upper part of the thickened posterior cortex and was directed towards the apex of the angulation.

Right TIBIA. Serial sections were unfortunately not available. Examination of sections showed the same abnormalities as those of the femur. The metaphyseal region was normal.

LeFT FibUla (Fig. 5). This showed a similar degree of 


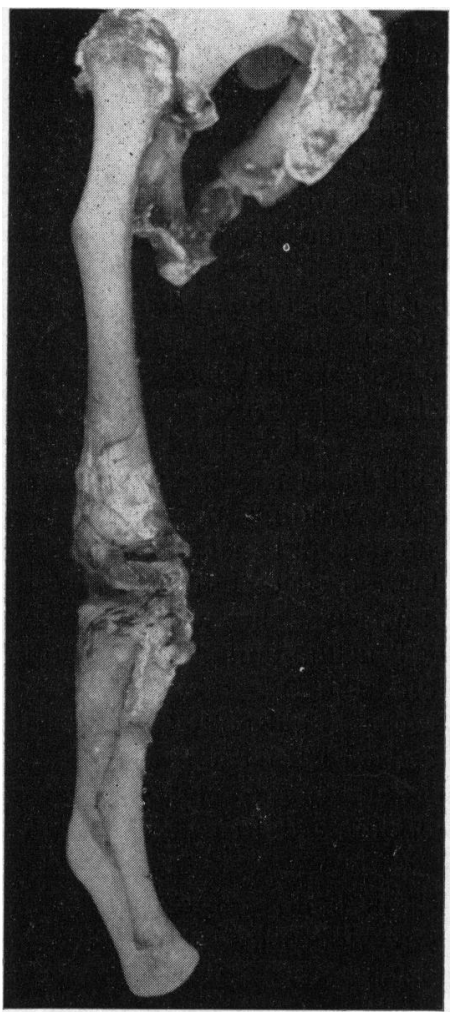

FIG. 5.

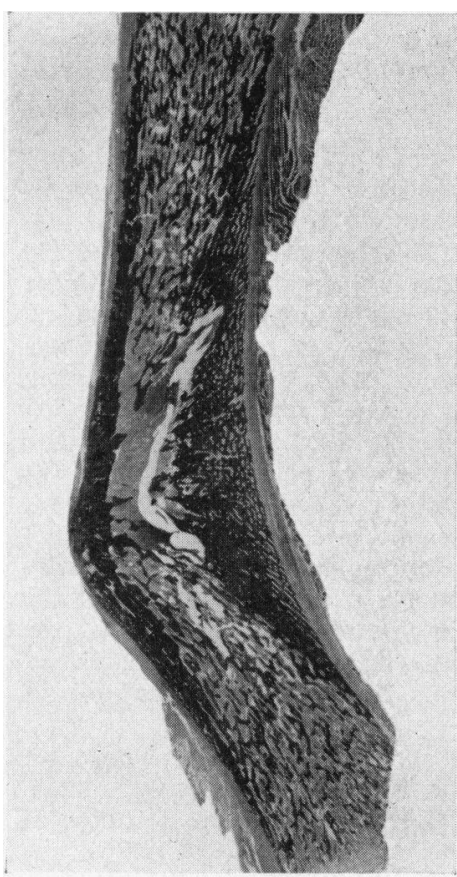

FIG. 6.

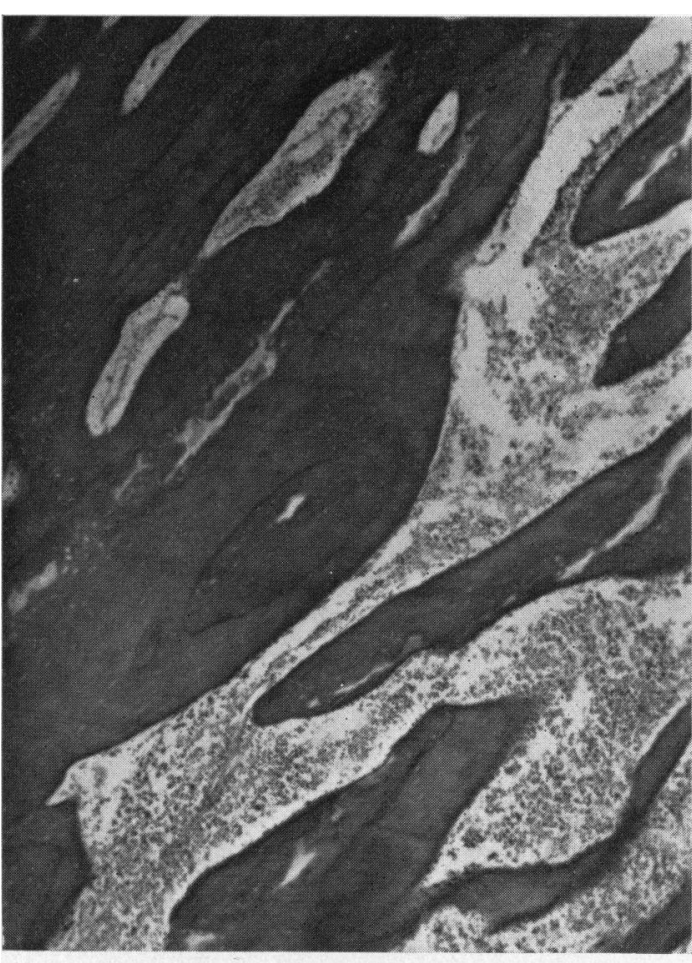

FIG. 7.

FIG. 5.-Lateral view of left lower limb showing angulations.

FIG. 6.-Section through angulation of femur showing thickened posterior cortex, radiating trabeculae and displaced medullary cavity. (H. and E. $\times 2 \frac{1}{2}$ )

FIG. 7.-Section of anterior cortex of femur immediately above angulation showing lines of irregular density, cartilage cores in trabeculae and areas of primitive bone. (H. and E. $\times 48$ ).

Fig. 8.-Abnormal scapula on right; scapula from normal full-term foetus on left. Absence of infraspinous portion of blade is obvious. Note poorly developed spine. Acromion process is absent and coracoid process is clearly seen.

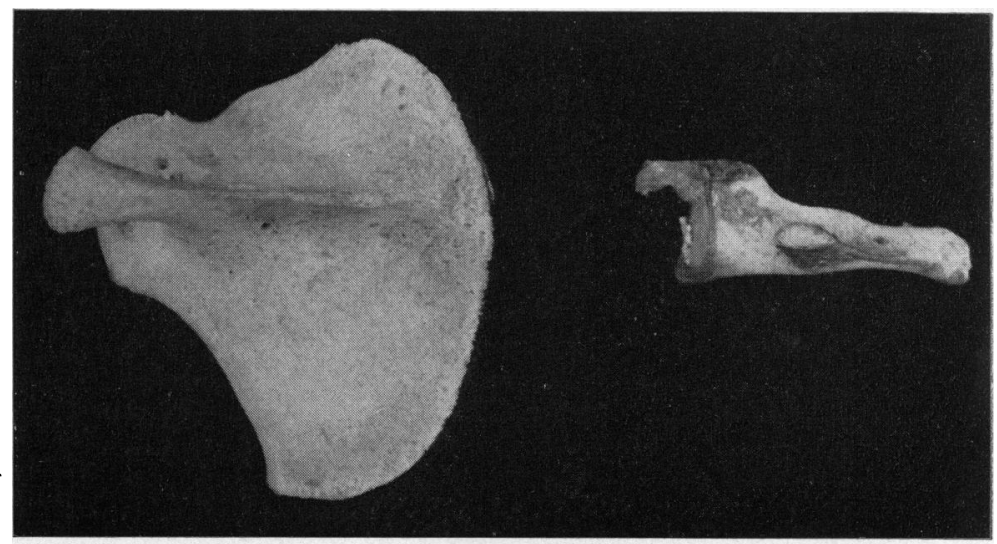

FIG. 8. 
angulation, the apex of which was slightly proximal to that of the tibia. The apex was not attached to the skin dimple. Sections showed that the architecture of the bone was similar to that of the tibia. The only nutrient artery entered the middle of the shaft posteriorly and ran in the upper radiating trabeculae towards a point just below the angulation.

Clavicles. These were normal.

SCAPUlaE (Fig. 8). These were very small, especially the blades, which were virtually absent below the level of the spines and were not represented by cartilage.

Humerus. A small spine projected from the posterolateral aspect of the shaft just above its midpoint. This spine marked the summit of a slight postero-lateral angulation of the shaft.

Section showed that the cortical bone was thickened to form the spine. Below the level of the spine, the cortex on the antero-medial aspect was thickened and composed of trabeculae radiating towards the spine. The nutrient artery ran in the upper border of the thickened cortex towards a point below the spine.

RADIUS AND UlNA. These were normal. Each bone showed two canals for the nutrient arteries.

\section{Discussion}

Multiple bowing of the long bones is not necessarily a fatal condition. Caffey (1947) records three cases in living children, and Bound, Finlay and Rose (1952) reported this condition in a child aged 5 months, in whom they noted the easy assumption at this age of the presumed foetal position in which each foot compressed the opposite thigh. Abnormalities accompanying the bowing have been noted by Williams (1943). In a case involving bowing of the femora and anterior angulation of the tibia, he noted incomplete ossification of the body and hypoplasia of the neural arch and spinous processes of the fifth cervical vertebra. In one of the cases of multiple bowing reported by Bound et al., there was micrognathia, cleft palate, plagiocephaly and a marked pigeon-breast deformity. Caffey, on the other hand, found no evidence of other deformities or other skeletal disease in his cases. Angle (1954), in a newly born premature infant, found no radiological evidence of other skeletal deformity except multiple bowing. In the present case the deformities of particular interest, other than the bowing, are those related to the scapula, the vertebrae and the pelvis. Angle (1954) stated that the most widely accepted hypothesis was the mechanical theory. This held that the curvatures in the bones were mechanical in origin resulting from the moulding of long bones over other parts by centripetal forces. Murray (1936) reproduces a photograph of an angulated tibia from a chondrodystrophic chick. The photograph shows bony trabeculae radiating from the concave aspect of the shaft towards the apex of the angulation. Murray attributes the formation of radiating trabeculae to normal ossification, occurring in a cartilage model pathologically soft and unable to resist bending stresses.

In the present case the long bones of the lower limbs were severely affected, the tibia and fibula being more deformed than the femur. The humerus was only slightly deformed and was atypical in that the spine lay above the level of the angulation. This spine might have been interpreted as a secondary marking caused by a fibrous attachment; such markings are not, however, normally present at birth. The slight angulation and cortical thickening on the concave aspect suggest that the humerus was affected in the same way as the lower limb bones but to a lesser extent.

The abnormality of the pelvic brim was similar to that described by Robert (1842), in which there was symmetrical transverse contraction with maldevelopment of the lateral masses of the sacrum and synostosis of the sacro-iliac joint. Robert's pelvis was transversely narrow at all levels, whereas the pelvis of this foetus was transversely narrow at the inlet only and very wide at the outlet. In this foetus the lateral masses of the sacrum were abnormal though the sacro-iliac joints were normal. Little (1958), who described a case of Robert's pelvis, could only find nine reported cases, it being doubtful if all these were indeed true examples of this type of pelvis. The abnormality of the sacrum and the pelvic brim in this case supports the theory that Robert's pelvis is primarily a congenital defect of the lateral masses of the sacrum.

Two-headed first ribs, like congenital dislocation of the hip, are fairly common as isolated abnormalities and these defects need not be further considered.

The abnormalities noted in this foetus were confined to tissues arising from mesoderm. With the exception of the cleft palate and unexplained hydronephrosis, these abnormalities were limited to that part of the ske'eton ossified in cartilage, though not all bones ossified in cartilage were abnormal, the radius and ulna being notable exceptions. We have tried to answer the following questions:

(1) Was the Original Cartilage Model of the Skeleton Normal? The disproportionately small size of the chondrocranium and of the lateral masses of the sacrum, the spina bifida and the almost complete absence of the cartilaginous precursor of the infraspinous portion of the blade of the scapula indicate either that, in these sites at least, a normal cartilaginous model had failed to develop, or that 
having developed, it was partly resorbed. The 'membranous' condition of parts normally cartilaginous at birth, i.e. the pedicles of some vertebrae and the laminae of others, and of the conjoint ramus is not necessarily significant since these parts have a high surface/mass ratio and maceration would therefore proceed more rapidly in these than in other parts. These membranous pedicles and laminae were nevertheless not ossified as they should have been. One possible reason for this could have been an abnormality of the cartilage.

We conclude that the cartilaginous axial skeleton was not normal and hence abnormality of the cartilaginous skeleton of the angulated limb bones cannot be excluded. As chondrification begins just after the end of the first month and is widespread by the end of the second month of gestation, the abnormality could have originated at this early period. Evidence bearing on the normality or abnormality of the precartilage condensations was naturally not available.

(2) Was the Process of Ossification of the Cartilage Models of Angulated Bones Normal? (a) Gross structure: Thickening of the cortex on the concave side of the angulated long bones was described by Angle (1954). He described the trabeculae of this cortex as radiating towards the apex of the curvature. It is universally accepted that the cortex of a long bone is very thin over the ends but becomes thick towards the middle of the shaft. Each bone of the six normal foetuses sectioned showed this cortical thickening on the concave aspect of the shaft, a thickening composed of trabeculae converging towards the convexity of the shaft. In every case the nutrient artery or arteries reached the medullary cavity by running between these trabeculae. In longitudinal sections of normal bones the trabeculae formed a triangle, the base of which was along the concave aspect of the shaft, and the apex of which was truncated by the medullary cavity. This triangle was present in both the abnormal and the normal bones. The abnormality in the angulated bones was not the presence of the radiating trabeculae but rather the shortness of the base of the triangle so formed.

The angulated bones showed normal modelling, the metaphysis being expanded and the cartilaginous epiphysis of normal shape. If the angulation of the shafts had been artificially corrected by removing a wedge from the convex side and resetting the upper and lower fragments, the resultant shaft would still have been abnormal because the shaft above and below the angulation was unduly straight and lacked the gentle curvature of the normal bone. Indeed, the angulated portion contained the whole longitudinal curvature of the shaft concentrated into a short length of bone, and the apex of the angulation pointed in the direction of the normal curvature.

The angulation of the long bones was symmetrical in degree, position and direction. We offer the following hypothesis. The primary centre of ossification in a long bone lies near the centre of the cartilage model. The more rapid growth in length of the growing end of the bone results in the gradual displacement of the site of the primary centre of ossification away from the growing end of that bone. The growing ends of the long bones are those at the wrist, shoulder and knee, except in the case of the fibula where it is probably situated in the lower end. Thus the site of the original primary centre of ossification would lie symmetrically in the upper parts of the shafts of the femora, in the lower parts of the shafts of the tibiae, a little higher in fibula than in tibia and in the lower part of the shaft of the humerus. These positions correspond approximately to the sites of angulation (Fig. 5) in the long bones in the present case. In the case of each of these bones the nutrient artery was directed through the thickened bone on the concave side of the angulation and, except in the humerus, towards the apex of the angulation. The abnormal situation and direction of the nutrient artery of the femur was probably of no significance. Indeed, the first two of six full-term normal femora, with which the angulated femur was compared, also showed the same anomaly of the canal for the nutrient artery.

(b) Histological examination of the bones was unfortunately limited, but the appearance of the femur and tibia showed that the epiphyseal and metaphyseal regions were normal, in sharp contrast to the shaft. The bony trateculae in the mid-shaft contained cartilaginous cores. This abnormally situated cartilage could have represented either a new formation of cartilage or a persistence of original cartilage. This latter hypothesis is unlikely as the medullary cavity was not abnormally small and all the bone developed in the original cartillaginous shaft must have been absorbed as the medullary cavity developed. Moreover, some subperiosteal trabeculae (i.e. those most recently formed and normally ossified without an intervening cartilaginous stage) showed cartilaginous cores. These observations suggest that the cartilaginous cores were a new formation. The lack of osteoblasts and osteoclasts noted may have been partly accounted for by the poor preservation of the material sectioned. This lack of cells, especially osteoclasts, was almost complete in the shaft and not evident in the metaphysis which was subjected to the 
same treatment. The lack of active bone resorption in the region of the spines of the long bones showed that ossification was not proceeding normally in this region at birth.

We conclude that ossification in the shafts of the femur and tibia was abnormal, and that the angulation occurred at approximately the site of the original centre of ossification which develops during the second month of gestation.

(3) Was the Cause of the Abnormality Extrinsic (Mechanical) or Intrinsic? Browne (1936) has stated that hydramnios is commonly associated with congenital, mechanically induced abnormalities, and that sparing of the forearm is associated with its protection from pressure by the head in the foetal position. The present foetus showed sparing of the forearm and there was also an associated hydramnios. The association of spina bifida in the second to the sixth cervical vertebrae with premature synostosis in the laminae of the seventh cervical and the first thoracic vertebrae is difficult to explain on a mechanical basis. Likewise, it is difficult to explain the abnormality of the scapula and sacrum on this basis. Any deforming force would have to be symmetrically applied in order to produce bilateral symmetrical deformities and it would also need to be maintained for a considerable period of time, i.e. from at least the second month onwards. Even had the angulation been produced by mechanical factors, the bone, if normal, ought to have shown some tendency to remodel to its normal form. There was no evidence whatsoever of bone resorption in the region of the spine.

We conclude that mechanical factors alone could not account for all the skeletal abnormalities present in this case, and an intrinsic abnormality of ossification in the angulated bones must have been present.

(4) What might be the Nature of this Intrinsic Defect? We suggest that the defect might have been located in the perichondrium and periosteum, although the state of the skeleton after maceration and section was unfit for investigations to prove or refute this theory. Ham (1957) states:

'The appearance of capillaries in the perichondrium profoundly affects the differentiation of the relatively undifferentiated cells of its inner layer. For, instead of continuing to develop into chondroblasts and chondrocytes, they, in the presence of capillaries, begin to differentiate into osteoblasts and osteocytes, with the result that a thin layer or shell of bone is soon laid down around the model. Since perichondrium thereafter covers bone tissue, its name is changed from perichondrium to periosteum. ... Indeed, the cells of the inner layer of the periosteum retain their ability to differentiate into chondroblasts and to form cartilage even into adult life. This is easily demonstrated in the repair of broken bones.'

There are thus two essential elements in the perichondrium, vascular and cellular. The perichondrium forms the perichondrial sleeve and gives rise to the periosteal bud which invades the cartilage model to form the primary centre of ossification.

Poor vascularization of the perichondrium of the angulated long bones, especially in the region of the periosteal bud, the capillaries of which become the nutrient artery, could account for the angulations being in the positions of the primary centres of ossification. Poor vascularization could also account for the presence of cartilage cores in some of the trabeculae of the angulated long bones. The normal forearm bones could then be associated with the presence of multiple vascular foramina which were observed in these bones and which probably represented a more adequate blood supply. The apparent normality of the metaphyseal and epiphyseal regions of the angulated bones could also be due to a more adequate blood supply towards the ends of the bones, such a blood supply being derived from the multiple small periosteal vessels.

Deficiency in the other (cellular) element of the perichondrium could be held to account for the paucity of bone cells in the angulated shafts.

Lacroix (1951) suggests that movement of the periosteal sleeve influences the arrangement of the subperiosteal trabeculae of a bone. In an angulated bone such failure of movement, localized to the concave side, might account for the angulation and the trabecular arrangement noted in this and in previously reported cases. This local failure, however, would not necessarily explain the abnormal straightness of the shaft above and below the angulation.

In this foetus we suggest that a perichondrium deficient in both cellular and vascular elements could have caused all the skeletal abnormalities.

\section{Summary}

A detailed examination has been made of a stillborn foetus showing multiple bowing of the long bones. In addition to the bowing of the bones other defects were found and those of particular interest were in the scapula, vertebrae and pelvis.

As a result of the studies on this case the following conclusions were reached:

(1) The cartilaginous axial skeleton was abnormal. The cartilaginous skeleton of the angulated bones may likewise have been abnormal.

(2) Ossification in the angulated long bones was abnormal. 
(3) The abnormality of ossification in the long bones was intrinsic in nature and situated approximately in the position of the primary centre of ossification.

(4) The basic disorder underlying the angulation was probably a deficiency in both vascular and cellular elements of the perichondrium.

We wish to thank Professor G. J. Romanes, Professor R. W. B. Ellis, Professor G. L. Montgomery and Professor L. H. Wells for their helpful criticism. We are grateful to Dr. J. B. King and Dr. W. Macleod for radiographs and to Dr. Nora Campbell for the drawings.
We also wish to thank Mr. T. C. Dodds and Miss C. Brydon for photography.

\section{REFERENCES}

Angle, C. R. (1954). Pediatrics, 13, 257. Bound, J. P., Finlay, H. V. L. and Rose, F. C. (1952). Arch. Dis.

Browne, D.'(1936). Proc. roy. Soc. Med., 29, 1409.

Caffey, J. (1947). Amer. J. Dis. Child., 74, 543.

Ham, A. W. (1957). Histology, 3rd ed., p. 278. Lippincott, Philadelphia.

Lacroix, P. (1951). The Organization of Bones, trans. S. Gilder, p. 67. Churchill, London.

Little, E. W. (1958). J. Obstet. Gynaec. Brit. Emp., 65, 465.

Murray, P. D. F. (1936). Bones: A Study of the Development and Structure of the Vertebrate Skeleton, p. 34. University Press, Cambridge.

Robert, F. (1842). Beschreibung eines im höchsten Grade querverengten Beckens. Karlsruhe.

Williams, E. R. (1943). Brit. J. Radiol., 16, 371. 